\title{
Preoperative tumor size measurement in breast cancer patients: which threshold is appropriate on computer-aided detection for breast MRI?
}

Sung Eun Song ${ }^{1}$, Bo Kyoung Seo ${ }^{2 *}$, Kyu Ran Cho ${ }^{1}$, Ok Hee Woo ${ }^{3}$, Eun Kyung Park ${ }^{2}$, Jaehyung Cha ${ }^{4}$ and Seungju $\operatorname{Han}^{5}$

\begin{abstract}
Background: Computer-aided detection (CAD) can detect breast lesions by using an enhancement threshold. Threshold means the percentage of increased signal intensity in post-contrast imaging compared to precontrast imaging. If the pixel value of the enhanced tumor increases above the set threshold, CAD provides the size of the tumor, which is calculated differently depending on the set threshold. Therefore, CAD requires the accurate setting of thresholds. We aimed to compare the diagnostic accuracy of tumor size measurement using MRI and CAD with 3 most commonly used thresholds and to identify which threshold is appropriate on CAD in breast cancer patients.

Methods: A total of 130 patients with breast cancers (80 invasive cancers and 50 ductal carcinoma in situ [DCIS]) who underwent preoperative MRI with CAD and surgical treatment were included. Tumor size was manually measured on first contrast-enhanced MRI and acquired by CAD using 3 different thresholds (30,50, and 100\%) for each tumor. Tumor size measurements using MRI and CAD were compared with pathological sizes using Spearman correlation analysis. For comparison of size discrepancy between imaging and pathology, concordance was defined as estimation of size by imaging within $5 \mathrm{~mm}$ of the pathological size. Concordance rates were compared using Chi-square test.

Results: For both invasive cancers and DCIS, correlation coefficient rho $(r)$ between tumor size on imaging and pathology was highest at CAD with 30\% threshold, followed by MRI, CAD with 50\% threshold, and CAD with 100\% threshold (all $p<0.05$ ). For invasive cancers, the concordance rate of $72.5 \%$ at CAD with $30 \%$ threshold showed no difference with that of $62.5 \%$ at MRI $(p=0.213)$. For DCIS, the concordance rate of $30.0 \%$ at CAD with $30 \%$ threshold showed no difference with that of $36.0 \%$ at MRI $(p=0.699)$. Compared to MRI, higher risk of underestimation was noted when using CAD with $50 \%$ or $100 \%$ threshold for invasive cancers and when using CAD with $100 \%$ threshold for DCIS.

(Continued on next page)
\end{abstract}

\footnotetext{
*Correspondence: seoboky@korea.ac.kr; seoboky@gmail.com

${ }^{2}$ Department of Radiology, Korea University Ansan Hospital, Korea University College of Medicine, 123 Jeokgeum-ro, Danwon-gu, Ansan-si, Gyeonggi-do 15355, Republic of Korea

Full list of author information is available at the end of the article
}

(c) The Author(s). 2020 Open Access This article is licensed under a Creative Commons Attribution 4.0 International License, which permits use, sharing, adaptation, distribution and reproduction in any medium or format, as long as you give appropriate credit to the original author(s) and the source, provide a link to the Creative Commons licence, and indicate if changes were made. The images or other third party material in this article are included in the article's Creative Commons licence, unless indicated otherwise in a credit line to the material. If material is not included in the article's Creative Commons licence and your intended use is not permitted by statutory regulation or exceeds the permitted use, you will need to obtain permission directly from the copyright holder. To view a copy of this licence, visit http://creativecommons.org/licenses/by/4.0/ The Creative Commons Public Domain Dedication waiver (http://creativecommons.org/publicdomain/zero/1.0/) applies to the data made available in this article, unless otherwise stated in a credit line to the data. 
(Continued from previous page)

Conclusion: For CAD analysis, 30\% threshold is the most appropriate threshold whose accuracy is comparable to manual measurement on MRI for tumor size measurement. However, clinicians should be aware of the higher risk of underestimation when using CAD with 50\% threshold for tumor staging in invasive cancers.

Keywords: Breast neoplasms, Magnetic resonance imaging, Neoplasm staging

\section{Introduction}

Preoperative evaluation of tumor size is important for appropriate surgical planning in breast cancer patients, particularly when planning in breast-conserving surgery. Negative margins can affect the risk of local recurrence $[1,2]$, which is $2-3$ times higher when associated with a positive margin than with a negative margin [3]. Multimodal breast imaging modalities are used for the preoperative assessment of tumor size and magnetic resonance imaging (MRI) is recognized as the most accurate imaging modality [4-8]. However, MRI had a substantial risk of overestimation, which can lead to upstaging of the type of surgery [9-11]. In addition, interand intra-observer variations when measuring tumor size are another drawback of MRI [12].

Breast evaluation using MRI takes considerable time and experience for image processing and manual kinetic curve analysis. In addition, breast MRI has a low specificity and a high false positive rate [13]. To overcome the limitations of breast MRI and to provide an easier way of interpreting enhancement characteristics, computer-aided detection (CAD) was developed and has been widely used since 2008 [13]. It provides quantitative tumor information such as tumor size, angio-volume and kinetic curve analysis, and improves interpretation efficiency of radiologists by speeding up image processing and analysis. Moreover, it improves the specificity of MRI by determination of benign and malignant lesions using kinetic curve assessment. For accurate use of CAD, setting of an enhancement threshold is important because CAD detects breast lesions by using an enhancement threshold. Threshold means the percentage of increased signal intensity in post contrast-enhanced imaging compared to pre-contrast imaging. If a pixel value of enhancing tumors increases above a set threshold, CAD assigns a specific color to each pixel of enhancing tumors. When the tumor have presence of a color overlay on the angio-map, CAD provide the calculated size of the enhancing tumor [13]. Low threshold values can yield many false-positive pixel predictions and overestimate the tumor size, whereas high threshold values can limit the sensitivity when showing the full lesion size and underestimate the tumor size [14]. Therefore, the appropriate use of $\mathrm{CAD}$ requires the accurate setting of thresholds.

To balance the sensitivity and specificity and to discriminate benign from malignant breast lesions, the most appropriate threshold for CAD was suggested as $50-60 \%$ $[15 ; 16]$. Therefore, most studies using CAD use the $50 \%$ threshold [15-20]. However, in our clinical practice, we have found that the extent of enhancing tumor analyzed with CAD can be differently calculated depending on the set threshold. For instance, when using the 100\% threshold on $\mathrm{CAD}$, calculated tumor sizes were much smaller than those using the $30 \%$ threshold or tumors were not detected by CAD because tumors couldn't increase above the $100 \%$ threshold. This issue has been raised by a previous research insisting that CAD can't replace the radiologist's assessment because it can cause negative enhancement of breast tumors [20].

Therefore, the purposes of this study were to compare tumor size measurement using MRI and CAD with the 3 most commonly used thresholds with pathological tumor size and to identify which threshold is appropriate on CAD for preoperative tumor size measurement in invasive cancers and DCIS.

\section{Materials and methods \\ Patients}

This retrospective study was performed with institutional review board approval. Between August 2008 to June 2012, we identified 160 consecutive breast cancer patients who underwent preoperative breast MRI and were processed by CAD before surgery in our institution. We excluded 39 patients (a) who underwent neoadjuvant chemotherapy before surgery $(n=13)$, (b) had multifocal or multicentric tumors that were clearly separated from each other at MRI $(n=10)$, (c) had no angiomaps because CAD did not manage large breast with matrix sizes greater than $512 \times 512 \times 256(n=8)$, or (d) had no exact tumor size on pathology report $(n=8)$. A total of 121 patients met these criteria and 9 patients had bilateral breast cancers. A total of 130 breast cancers-80 invasive cancers and 50 ductal carcinoma in situ (DCIS) - were included. The patients' mean age was 51 years (range, $31-79$ years).

We reviewed medical records to identify the presence of local recurrence after treatment. For determining molecular subtypes and adjuvant systemic chemotherapy, immunohistochemistry was done for all cancers. Local recurrence, defined as recurrence in the ipsilateral breast or chest, was determined when histological type and receptor status was concordant between primary, and 
recurrent cancer. The last date of data collection was February 19, 2019. The median follow-up duration was 84.0 months (range, 5.9-99.2 months).

\section{MRI examination}

All breast MRI examinations were performed with the patients in the prone position using a 3.0-T MR imaging system (Achieva 3.0 T TX; Philips Healthcare, Best, the Netherlands) equipped with dedicated four-element SENSE-compatible breast surface coils (MRIDevices; InVivo Research, Orlando, Fla). Dynamic bilateral sagittal T1-weighted three-dimensional gradient echo images were acquired before and after contrast media injection with a total of six dynamic acquisitions. Twenty $\mathrm{ml}$ of $0.5 \mathrm{mmol} / \mathrm{ml}$ Gadodiamide (Omniscan; NycomedAmersham, Princeton, NJ, USA) was injected intravenously followed by a $20-\mathrm{ml}$ saline flush at the rate of 2.0 $\mathrm{ml} / \mathrm{sec}$. Five contrast-enhanced images were obtained at $60,120,180,240$, and $300 \mathrm{~s}$. Axial and coronal 3D reconstruction images with maximum intensity projection were obtained (TR/TE 3.4/1.3, flip angle $10^{\circ}$, field of view $320 \times 320 \mathrm{~mm}^{2}$, acquired voxel size $0.91 \times 0.91 \times$ $2.00 \mathrm{~mm}^{3}$, reconstructed voxel size $0.83 \times 0.83 \times 1.00$ $\mathrm{mm}^{3}$ ).

\section{CAD system}

For CAD analysis, all MRI images were transferred to a commercially available CAD system (CADstream, Confirma Inc. Bellevue, WA). This workstation compared pixel intensity values on the precontrast and first contrast-enhanced series to classify enhancement. If the pixel value increased above a set enhancement threshold, CAD assigned a specific color to each pixel of tumors. If a pixel value of tumor did not increase by the established threshold, no color enhancement was made on the tumor. When the tumor above the set threshold had a presence of color overlay on the angio-map, CAD semi- automatically calculated tumor size using coronal, axial, and sagittal images and provided the three-plane measured sizes (Fig. 1). Some areas outside the breast such as blood vessels were also colored on the angio-map, but CAD was trained to accurately recognize the area of enhancement of the breast and did not include it in tumor size measurements. For CAD analysis, we applied 3 thresholds (30, 50, and 100\%) which were most commonly used in clinical practice and acquired the tumor sizes calculated by CAD at each threshold (Figs. 2 and 3 ). Among three- plane measured sizes of tumors generated by $\mathrm{CAD}$, we selected the longest tumor size.

\section{Imaging evaluation}

On breast MRI, MRI lesion type and tumor size measurements were evaluated by 1 breast imaging radiologist (S.E.S., with 9 years' experience) who was blinded to the clinicopathologic information. The lesion type (mass or non-mass enhancement [NME]) was described according to the Breast Imaging Reporting and Data System Atlas for MRI [21]. The three-dimensional sizes of tumors were manually measured on the first contrastenhanced MRI using coronal, axial, and sagittal images in the same way as CAD and then, we selected the longest diameter of each tumor for comparison with tumor size by CAD.

\section{Pathology evaluation}

Of the 130 breast cancer cases, 67 underwent breastconserving surgery and 63 underwent a mastectomy. As soon as possible after surgery, the specimens were quickly delivered to the pathology department, sectioned, placed in $10 \%$ neutral buffered formalin, paraffin embedded, and stained with hematoxylin and eosin [22]. Along the vertical direction of the connecting line between the tumor and the nipple, sections were made every $5 \mathrm{~mm}$ for breast-conserving specimens and every

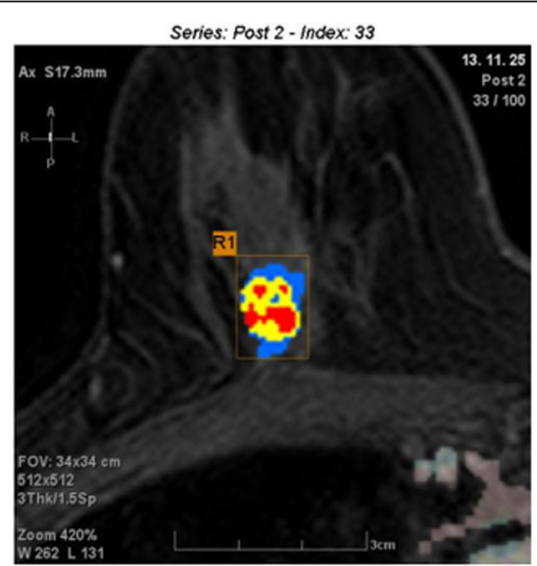

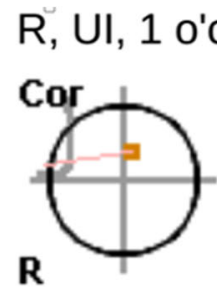

Diameters: $1.4 \times 0.89 \times 2.1 \mathrm{~cm}$ Angio Volume: $1.2 \mathrm{cc}$

Fig. 1 CAD angio-map showing how CAD provided the three-plane measured sizes using coronal, axial, and sagittal images 

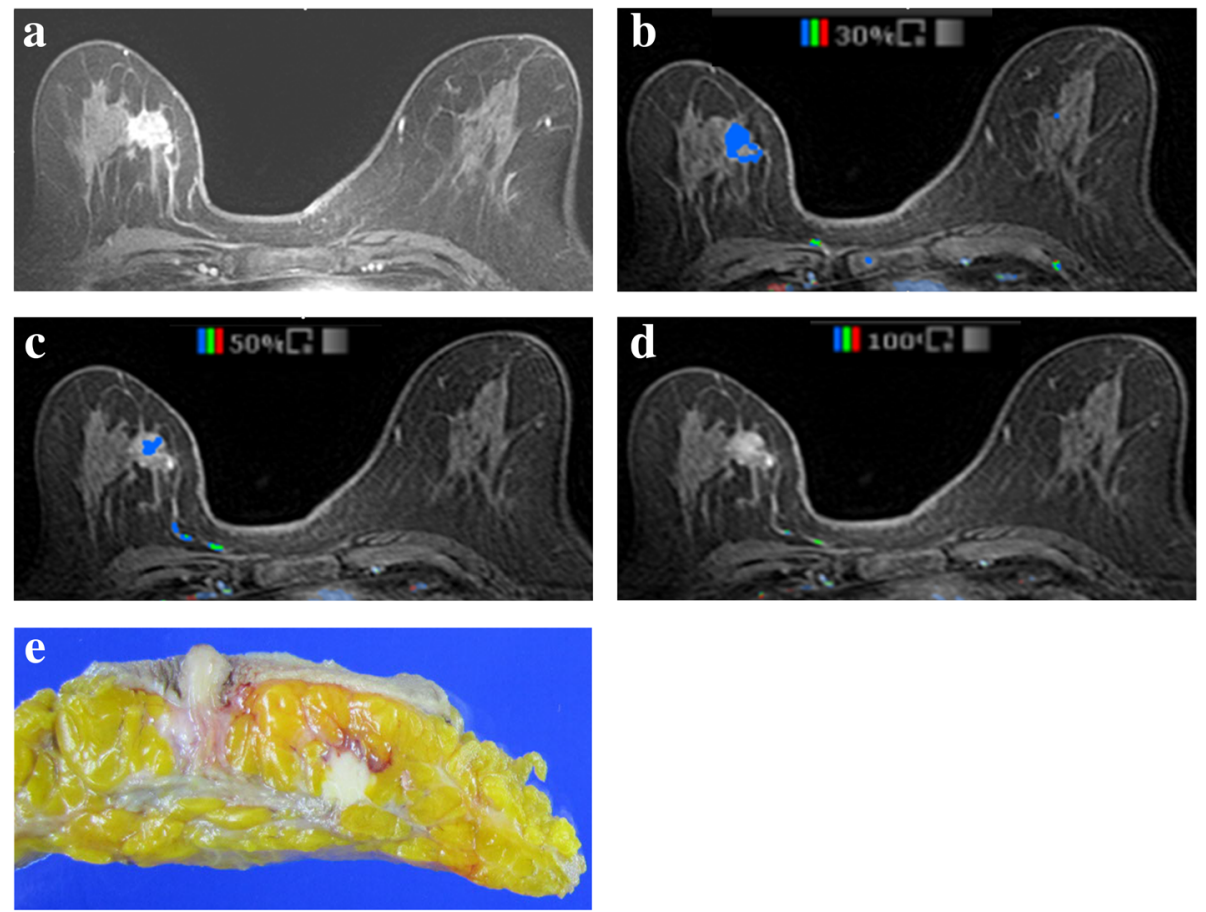

Fig. 2 MR images of a 73-year-old-woman with invasive ductal carcinoma. a Axial fat-suppressed dynamic contrast-enhanced T1-weighted image depicts a $19 \mathrm{~mm}$-sized irregularly shaped, irregularly marginated, heterogeneous enhancing mass at right middle-inner breast. $\mathbf{b}$ and $\mathbf{c}$ In the same image, the computer-aided detection system displayed the mass as the presence of color overlay that measured $17 \mathrm{~mm}$ at the $30 \%$ threshold and $9 \mathrm{~mm}$ at the 50\% threshold. d The enhancing mass was not detected at the $100 \%$ threshold. e Actual pathologic tumor size was $22 \mathrm{~mm}$ on gross specimen

$10 \mathrm{~mm}$ for mastectomy specimens. Pathological tumor size was recorded by experienced pathologists with 8 20 years' experience. For breast-conserving specimens, the largest size was determined by measuring the stained slides directly and fitting adjacent blocks containing the tumor. For mastectomy specimens, the largest size was measured by sampling the tissue with the largest sections [23]. For invasive cancers, the longest diameter of the invasive tumor alone and the total tumor size (both invasive and in situ carcinoma components) was recorded. For DCIS, the size of the in situ carcinoma components was assessed by counting the sections that included DCIS and by measuring the maximal diameter of the lesion on the mounted sections [23].

\section{Statistical analysis}

Spearman correlation analysis was used to calculate the linear association (correlation coefficient rho $[r]$ ) between tumor size measurement with MRI or CAD and pathological tumor sizes. A paired $t$ test was used to compare the mean difference in tumor size measurement on imaging and pathological tumor size.

To define concordance, underestimation, and overestimation, the size discrepancy was estimated as follows. The tumor size measured with MRI or CAD subtracted from the pathological tumor size. If the size discrepancy was $\leq \pm 5 \mathrm{~mm}$, the measured tumor size was classified as a concordance [24]. If the measured tumor size underestimated the pathological tumor size by $>5 \mathrm{~mm}$, the measured tumor size was classified as underestimation [24]. If the measured tumor size overestimated pathological tumor size by $>5 \mathrm{~mm}$, the measured tumor size was classified as overestimation [24]. The chi-square test was used to compare the proportions of underestimation, concordance, and overestimation for each CAD threshold with those obtained with manual measurement on MRI as the reference standard, and was also used to compare the local reccurence rate between invasive cancers and DCIS. These analyses were performed using SPSS Statistics for Windows, version 20 (IBM Corp.).

Test of significance for correlations was additionally done for comparing the statistical difference between the two independent correlations acquired from Spearman correlation analysis. Bland-Altman limits of agreements (LOA) were also used to compare the differences between the tumor sizes on MRI or CAD and the pathological tumor sizes (reference standard) versus their mean. These two analyses were done with $\mathrm{R}$ version 3.0.2; $P$ values $<0.05$ were considered significant. This retrospective study was approved by our institutional 

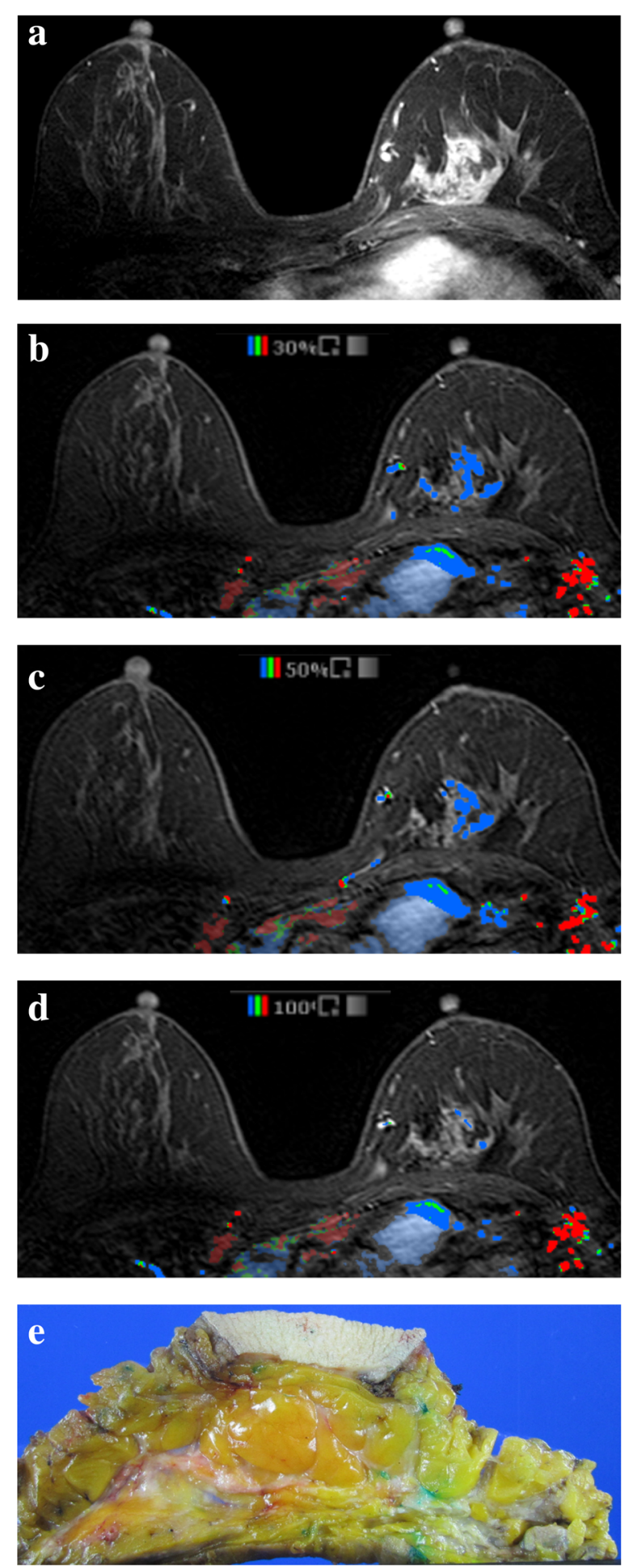

Fig. 3 MR images of a 54-year-old-woman with ductal carcinoma in situ. a Axial fat-suppressed dynamic contrast-enhanced T1-weighted image shows $43 \mathrm{~mm}$-sized regionally distributed non-mass enhancement in the left middle-inner breast. $\mathbf{b}$ - $\mathbf{d}$ In the same image obtained with a computer-aided detection system, the non-mass enhancement indicated by the presence of color overlay was measured as $34 \mathrm{~mm}$ at the $30 \%$ threshold, $24 \mathrm{~mm}$ at the 50\% threshold, and $21 \mathrm{~mm}$ at the 100\% threshold. e Actual pathologic tumor size was $38 \mathrm{~mm}$ on gross specimen review board, and the requirement for obtaining informed consent was waived.

\section{Results}

Lesions and follow-up

Pathologically, the most invasive cancers were invasive ductal carcinomas (73 of 80, 91.3\%), and the other cancers (7 of $80,8.7 \%)$ were 5 invasive lobular carcinomas, 1 invasive apocrine carcinoma, and 1 invasive cribriform carcinoma. Of the 80 invasive cancers, 72 (90.0\%) cancers had absent or focal DCIS and remaining 8 (10.0\%) had extensive DCIS. Of the 80 invasive cancers, 76 (95.0\%) were masses and the other 4 (5.0\%) were NME. Of the 50 DCIS, 25 (50.0\%) were masses and 25 (50.0\%) were NME.

For the 80 invasive cancers and the 50 DCIS cases, positive and negative enhancement at MRI and CAD with 3 different thresholds were summarized in Table 1 . Negative enhancement meant there was no enhancing areas in the breast. For the 80 invasive cancers, 1 case showed no enhancement at the 50\% threshold and 23 cases showed no enhancement at the 100\% threshold. For the 50 DCIS cases, 4 cases showed no enhancement at the $50 \%$ threshold and 21cases showed no enhancement at the $100 \%$ threshold.

Follow-up examination showed that local recurrence had occurred in 7 of the 130 cancers (5.4\%). The local recurrence was more frequent for the invasive cancers $(7.5 \%, 6$ of 80$)$ than for DCIS $(2 \%, 1$ of 50$)(p=0.249)$. The lesion type of the 7 cases of local recurrence were all masses. Among these 7 cases with local recurrence, all were MRI-concordant cases. However, 1 case was underestimated at both the 30 and 50\% thresholds of $\mathrm{CAD}$, and 3 cases were underestimated at the 100\% threshold of CAD.

\section{Correlation and concordance rate in the 80 invasive cancers}

For the 80 invasive cancers, the correlation coefficient was highest at CAD with $30 \%$ threshold $(r=0.808)$, followed by manual measurement on MRI $(r=0.778)$, CAD with 50\% threshold $(r=0.729)$, and CAD with $100 \%$ threshold $(r=0.386)$ (all $p<0.001)$ (Table 2).

The concordance rate of $72.5 \%$ at CAD with $30 \%$ threshold showed no difference with that of $62.5 \%$ at manual measurement on MRI $(p=0.213)$. However, underestimation rate of $35.0 \%$ at CAD with $50 \%$ threshold and that of $71.2 \%$ at CAD with $100 \%$ threshold were higher than that of $16.2 \%$ on manual measurement on MRI ( $p=0.036$, and $p<0.001$, respectively) (Table 3 ).

The Bland-Altman plot showed that the LOA of CAD with $30 \%$ threshold were narrower compared with those of the other imaging modalities (Fig. 4). 
Table 1 Positive and negative enhancement at MRI and CAD in 130 breast cancers

\begin{tabular}{|c|c|c|c|c|}
\hline 80 Invasive cancers & Manual measurement on MRI ${ }^{a}$ & CAD ${ }^{b}$ with $30 \%$ threshold & CAD with $50 \%$ threshold & CAD with $100 \%$ threshold \\
\hline Positive enhancement & $80(100.0) *$ & $80(100.0)$ & 79 (98.8) & $57(71.3)$ \\
\hline Negative enhancement & $0(0.0)$ & $0(0.0)$ & $1(1.2)$ & $23(28.7)$ \\
\hline Total Number & $80(100.0)$ & $80(100.0)$ & $80(100.0)$ & $80(100.0)$ \\
\hline 50 ductal carcinoma in situ & Manual measurement on MRI & CAD with $30 \%$ threshold & CAD with $50 \%$ threshold & CAD with $100 \%$ threshold \\
\hline Positive enhancement & $50(100.0)$ & $50(100.0)$ & $46(92.0)$ & $29(58.0)$ \\
\hline Negative enhancement & $0(0.0)$ & $0(0.0)$ & $4(8.0)$ & $21(42.0)$ \\
\hline Total Number & $50(100.0)$ & $50(100.0)$ & $50(100.0)$ & $50(100.0)$ \\
\hline
\end{tabular}

* Data are numbers of cancers with percentages in parentheses

${ }^{a} M R I$ magnetic resonance imaging, ${ }^{b} C A D$ computer-aided detection

\section{Correlation and concordance rate in the 50 DCIS cases}

For the 50 DCIS cases, the correlation coefficient was highest at CAD with $30 \%$ threshold $(r=0.659)$, followed by manual measurement on MRI $(r=0.620)$, CAD with $50 \%$ threshold $(r=0.600)$, and CAD with $100 \%$ threshold $(r=0.370)($ all $p<0.05)$ (Table 4$)$.

The concordance rates of $30.0 \%$ at CAD with $30 \%$ threshold and $34.0 \%$ at CAD with 50\% threshold showed no difference with that of $36.0 \%$ at manual measurement on MRI ( $p=0.699$ and $p=0.744$, respectively). However, underestimation rates of $76.0 \%$ at CAD with $100 \%$ threshold was higher than that of $32.0 \%$ on manual measurement on MRI $(p<0.001)$ (Table 5).

The Bland-Altman plot showed that the LOA of CAD with $30 \%$ threshold were narrower compared with those of the other imaging modalities (Fig. 5).

\section{Discussion}

Breast MRI is considered to be the most accurate imaging modality for preoperative tumor size measurement in breast cancer patients. However, it also has disadvantages such as over- or underestimation of the tumor size $[5,7,9-11,25,26]$ and inter- and intra-observer variations [12]. To overcome these shortcomings, CAD is used as an alternative for quantifying the tumor size.
However, only a few studies have investigated tumor size measurement using CAD for invasive cancers [8, 27, 28]. Whereas two studies using the $50 \%$ threshold reported that CAD was feasible for preoperative staging [8] and for predicting the residual tumor size after neoadjuvant chemotherapy [28], another study using the $100 \%$ threshold concluded that CAD is less accurate [27]. These inconsistent results may reflect the use of different threshold settings, which are the most important parameter when using CAD. Previous studies have reported that the threshold should not be set at $<50 \%$ because of the risk of high false-positive rates and should not be set at $>60 \%$ because of the limited ability to identify the full lesion size [14, 15]. Hence, the commonly used threshold in clinical practice is $50 \%$, although the user can select the threshold from the range of 30 to $200 \%$ [8, 15-20, 27, 28]. To determine the appropriate thresholds for the use of CAD in preoperative tumor size measurement, further studies comparing the tumor size measurements using different thresholds for each type of tumor are required. To our knowledge, the present study is the first to assess the accuracy of tumor size assessment with MRI and CAD using 3 thresholds for both invasive cancers and DCIS.

Table 2 Tumor sizes and correlation coefficients in 80 invasive cancers

\begin{tabular}{|c|c|c|c|c|c|}
\hline & Pathology & $\begin{array}{l}\text { Manual measurement } \\
\text { on MRI }^{a}\end{array}$ & $\begin{array}{l}\text { CAD }^{\mathrm{b}} \text { with } 30 \% \\
\text { threshold }\end{array}$ & $\begin{array}{l}\text { CAD with } 50 \% \\
\text { threshold }\end{array}$ & $\begin{array}{l}\text { CAD with } 100 \% \\
\text { threshold }\end{array}$ \\
\hline Mean tumor size $(\mathrm{mm})$ ( $p$ value $\left.{ }^{*}\right)$ & $\begin{array}{l}22.47 \pm \\
13.79\end{array}$ & $22.77 \pm 12.69(0.726)$ & $\begin{array}{l}22.35 \pm 13.96 \\
(0.887)\end{array}$ & $\begin{array}{l}19.15 \pm 13.75(< \\
0.001)\end{array}$ & $\begin{array}{l}9.91 \pm 11.55(< \\
0.001)\end{array}$ \\
\hline Median tumor size (mm) & 20.00 & 20.00 & 18.00 & 15.00 & 6.50 \\
\hline Size range $(\mathrm{mm})$ & $5-85$ & $6-69$ & $5-68$ & $0-66$ & $0-63$ \\
\hline Correlation coefficient $\left(p\right.$ value ${ }^{\dagger}$ ) & & $0.778(<0.001)$ & $0.808(<0.001)$ & $0.729(<0.001)$ & $0.386(<0.001)$ \\
\hline $\begin{array}{l}\text { Test of difference between two independent } \\
\text { correlations }\left(p \text { value }{ }^{\dagger+}\right)\end{array}$ & & & $(0.921)$ & $(0.080)$ & $(<0.001)$ \\
\hline
\end{tabular}

* A paired t test was used to compare the mean difference in tumor size measurement with MRI or CAD and pathological tumor size

+ Spearman correlation analysis was used to calculate the correlation coefficient between tumor size measurements with MRI

or CAD and pathological tumor size

t† Test of difference between two independent correlations was used to test the difference between MRI and CAD with the $30 \%$ threshold or between MRI and CAD with the $50 \%$ threshold or between MRI and CAD with the $100 \%$ threshold

${ }^{\mathrm{a}} \mathrm{MRI}$ magnetic resonance imaging, ${ }^{\mathrm{b}} \mathrm{CAD}$ computer-aided detection 
Table 3 Concordance rates of MRI and CAD in 80 invasive cancers

\begin{tabular}{|c|c|c|c|c|}
\hline & Manual measurement on $\mathrm{MRI}^{\mathrm{a}}$ & CAD $^{\mathrm{b}}$ with $30 \%$ threshold & CAD with $50 \%$ threshold & CAD with $100 \%$ threshold \\
\hline Underestimation & $13(16.2)$ & $12(15.0)$ & $28(35.0)$ & $57(71.2)$ \\
\hline Concordance & $50(62.5)$ & $58(72.5)$ & $43(53.8)$ & $23(28.7)$ \\
\hline Overestimation & $17(21.3)$ & $10(12.5)$ & $9(11.2)$ & $0(0.0)$ \\
\hline Total Number & $80(100.0)$ & $80(100.0)$ & $80(100.0)$ & $80(100.0)$ \\
\hline$p$ valuet & & 0.213 & 0.036 & $<0.001$ \\
\hline
\end{tabular}

* Data are numbers of cancers with percentages in parentheses

t The chi-square test was used to compare the outcomes of CAD with that those of MRI as a reference standard

${ }^{a} M R I$ magnetic resonance imaging, ${ }^{b} C A D$ computer-aided detection

For invasive cancer, CAD with 30\% threshold showed the best performance outcomes for correlation coefficient, concordance rate, and underestimation rate. When using CAD with $50 \%$ or $100 \%$ threshold, underestimation rates were significantly higher than that of manual measurement on MRI. However, the underestimation rate of $15.0 \%$ at CAD with $30 \%$ threshold was similar to that of $16.2 \%$ at manual measurement on MRI. Taken together, these data suggest that CAD with $30 \%$ threshold is the most precise tool and is comparable to MRI without the risk of underestimation. In our study, the observation that the $100 \%$ threshold had a significantly higher underestimation rate was expected from previous studies that have reported limited sensitivity of the $100 \%$ threshold at CAD $[14,15]$. We also note that the $50 \%$ threshold which has been most widely used in clinical practice had a significantly higher underestimation rate of $35.0 \%$, which was more than twice as high as the $16.2 \%$ for MRI.

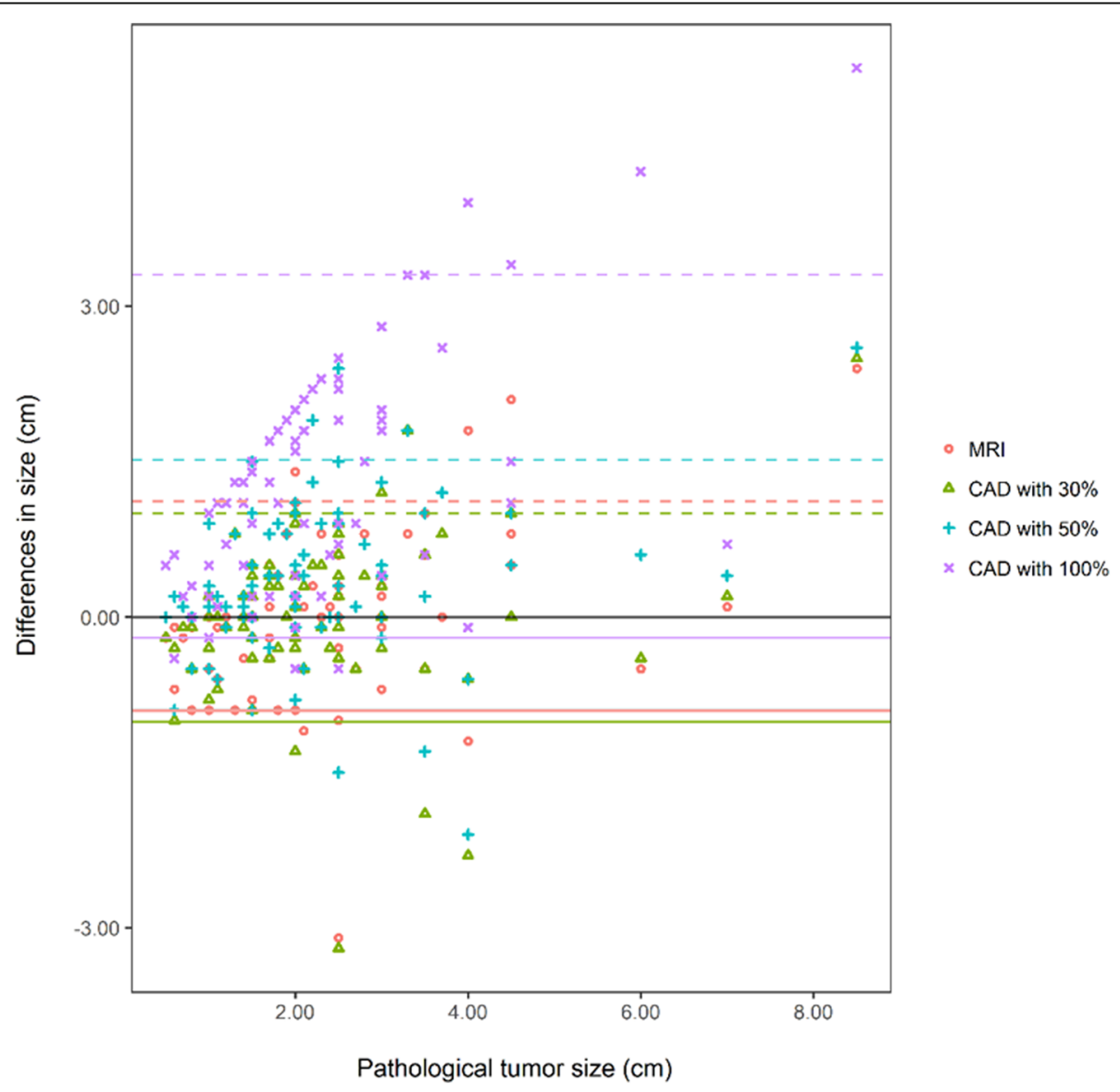

Fig. 4 The Bland-Altman plot for comparison of the differences between the tumor sizes on MRI or CAD and the pathological tumor sizes in 80 invasive cancers. Times indicates MRI size and pathological tumor size. Triangle indicates CAD with 30\% threshold. Cross indicates CAD with 50\% threshold. X mark indicates CAD with 100\% threshold. This plot shows that that the limits of agreements of CAD with $30 \%$ threshold are narrower compared with those of the other imaging modalities 
Table 4 Tumor sizes and correlation coefficients in 50 ductal carcinoma in situ

\begin{tabular}{|c|c|c|c|c|c|}
\hline & Pathology & $\begin{array}{l}\text { Manual measurement } \\
\text { on MRI }^{\text {a }}\end{array}$ & $\begin{array}{l}C A D^{b} \text { with } 30 \% \\
\text { threshold }\end{array}$ & $\begin{array}{l}\text { CAD with } 50 \% \\
\text { threshold }\end{array}$ & $\begin{array}{l}\text { CAD with } 100 \% \\
\text { threshold }\end{array}$ \\
\hline Mean tumor size $(\mathrm{mm})$ ( $p$ value*) & $\begin{array}{l}24.96 \pm \\
17.97\end{array}$ & $25.80 \pm 15.50(0.678)$ & $\begin{array}{l}27.06 \pm 17.00 \\
(0.297)\end{array}$ & $\begin{array}{l}21.54 \pm 17.45 \\
(0.118)\end{array}$ & $\begin{array}{l}8.28 \pm 12.29(< \\
0.001)\end{array}$ \\
\hline Median tumor size (mm) & 21.00 & 23.50 & 23.00 & 17.00 & 4.00 \\
\hline Size range $(\mathrm{mm})$ & $4-90$ & $4-78$ & $6-84$ & $0-81$ & $0-60$ \\
\hline Correlation coefficient $\left(p\right.$ value ${ }^{\dagger}$ ) & & $0.620(<0.001)$ & $0.659(<0.001)$ & $0.600(<0.001)$ & $0.370(0.002)$ \\
\hline $\begin{array}{l}\text { Test of difference between two independent } \\
\text { correlations }\left(p \text { value }{ }^{+t}\right)\end{array}$ & & & $(0.810)$ & $(0.890)$ & $(0.120)$ \\
\hline
\end{tabular}

* A paired $t$ test was used to compare the mean difference in tumor size measurement with MRI or CAD and pathological tumor size

+ Spearman correlation analysis was used to calculate the correlation coefficient between tumor size measurements with MRI

or CAD and pathological tumor size

tt Test of difference between two independent correlations was used to test the difference between MRI and CAD with the $30 \%$ threshold or between MRI and CAD with the $50 \%$ threshold or between MRI and CAD with the $100 \%$ threshold

${ }^{a} M R I$ magnetic resonance imaging, ${ }^{b} C A D$ computer-aided detection

MRI is increasingly used for the preoperative staging in DCIS $[7,25,29]$. Our correlation coefficients for MRI or for the $30 \%$ or $50 \%$ threshold at CAD were all within the range of 0.409 to 0.786 , as reported from previous studies [7, 29]. However, both the correlation coefficients and concordance rates were considerably lower for DCIS than for invasive cancers, and these low rates may reflect the inherent characteristics of DCIS $[9,25$, 30-32]. In terms of morphologic feature, NME rather than masses is the dominant feature of DCIS [25, 3032]. In a previous study of the factors influencing discordance of tumor size measurements [9], only NME significantly predicted discordance. Moreover, for kinetic feature, NME had less suspicious enhancement kinetics than mass lesions $[31,32]$. Therefore, we conclude that, for DCIS, tumor size measurement with CAD using the $30 \%$ or $50 \%$ threshold should be able to achieve equivalent accuracy in predictions as that using MRI; however, the results were inferior to those for invasive cancers probably because of the inherent characteristics of DCIS.

The issue of overestimation with the use of MRI to measure tumor size has been consistently raised. Although some studies have reported that MRI overestimates the pathological tumor size [5, 7, 33], other studies have reported that MRI underestimates the pathological size $[25,26]$. In the MRI analysis of our study, overestimation was more frequent than underestimation in the invasive cancers, and both overestimation and underestimation took place with similar probabilities in the DCIS cases. Interestingly, the overestimation rate decreased and concordance rate increased for invasive cancers when analyzed using the 30\% threshold at CAD. This may reflect the interconnection between enhancement of high-risk lesions and proliferative disease associated with invasive tumors, which has also been suggested as a reason for overestimation by MRI, are not as well recognized by CAD because they do not enhance above a set threshold of CAD [34]. Accordingly, the application of CAD with $30 \%$ threshold may help to decrease the high overestimation rate of invasive breast cancers when analyzed using MRI.

Underestimation is also great importance because it may lead to inadequate treatment, which can influence the risk of local recurrence. In our study, we observed negative enhancement of tumors at CAD with $50 \%$ or $100 \%$ thresholds. Not only negative enhancement but also underestimation of tumor sizes by CAD can limit the use of $\mathrm{CAD}$ in the preoperative tumor staging because it can affect the local recurrence. In the present study, 7 cases of local recurrence were found on followup; of these 7 cases, 3 cases had been underestimated at the $100 \%$ threshold of CAD and 1 had been

Table 5 Concordance rates of MRI and CAD in 50 ductal carcinoma in situ

\begin{tabular}{lllll}
\hline & Manual measurement on MRI ${ }^{a}$ & CAD $^{\text {b }}$ with $30 \%$ threshold & CAD with 50\% threshold & CAD with 100\% threshold \\
\hline Underestimation & $16(32.0)^{*}$ & $15(30.0)$ & $20(40.0)$ & $38(76.0)$ \\
Concordance & $18(36.0)$ & $15(30.0)$ & $17(34.0)$ & $9(18.0)$ \\
Overestimation & $16(32.0)$ & $20(40.0)$ & $13(26.0)$ & $3(6.0)$ \\
Total number & $50(100.0)$ & $50(100.0)$ & $50(100.0)$ & $50(100.0)$ \\
$p$ valuet & & 0.699 & 0.744 & $<0.001$ \\
\hline
\end{tabular}

* Data are numbers of cancers with percentages in parentheses

t The chi-square test was used to compare the outcomes of CAD with that those of MRI as a reference standard

${ }^{a} M R I$ magnetic resonance imaging, ${ }^{b} C A D$ computer-aided detection 


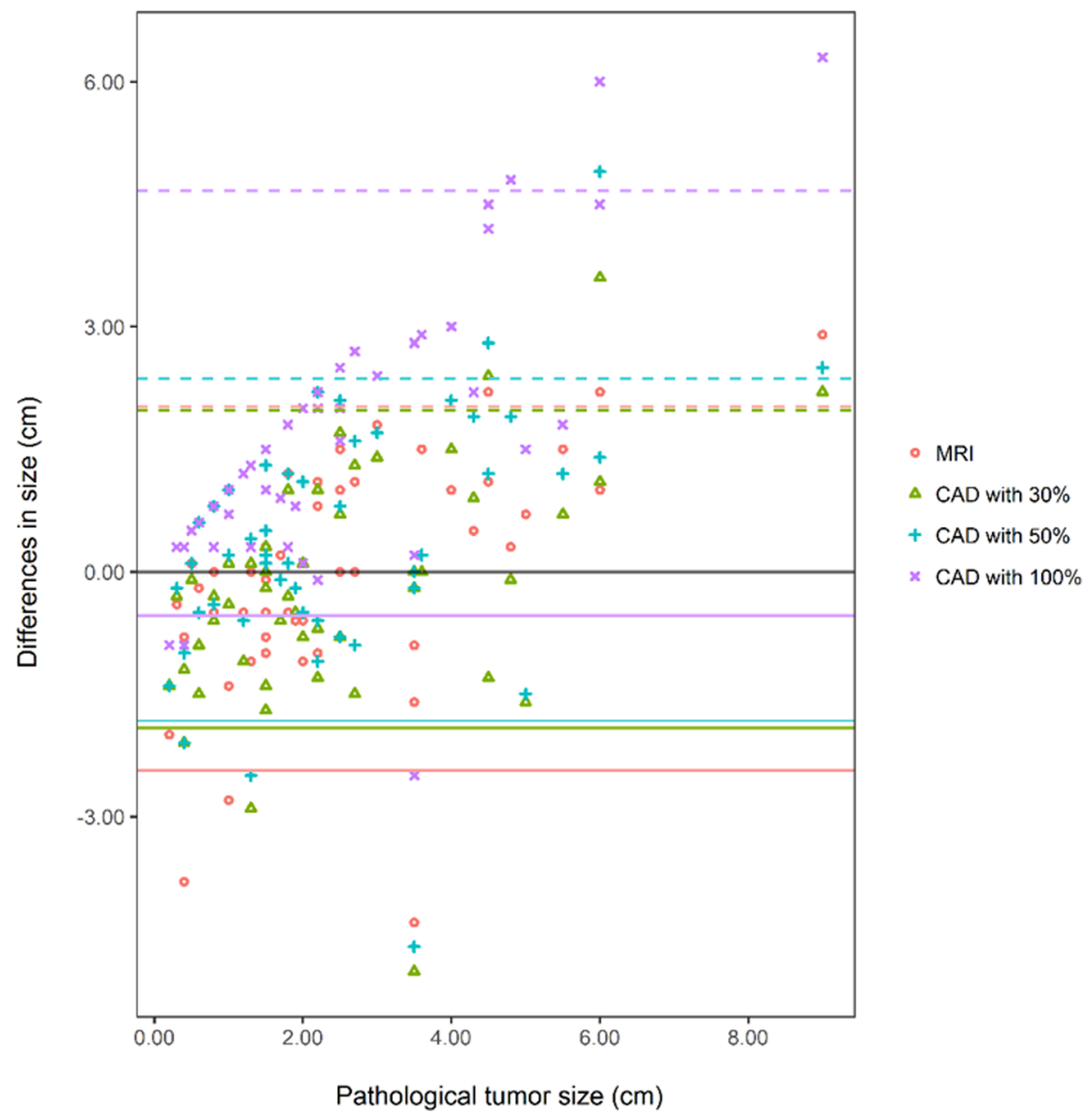

Fig. 5 The Bland-Altman plot for comparison of the differences between the tumor sizes on MRI or CAD and the pathological tumor sizes in 50 ductal carcinoma in situ. Times indicates MRI size and pathological tumor size. Triangle indicates CAD with 30\% threshold. Cross indicates CAD with 50\% threshold. X mark indicates CAD with 100\% threshold. This plot shows that that the limits of agreements of CAD with 30\% threshold are narrower compared with those of the other imaging modalities

underestimated at both the 30 and $50 \%$ thresholds. Therefore, when using CAD in preoperative tumor size measurement, it is essential to be aware of the higher risk of underestimation which may influence the local recurrence.

Although our preliminary results are promising, our study has several limitations. First, this was a retrospective review of records from a single institution. Second, the tumor extent was measured manually on MRI by only 1 experienced breast radiologist, and the lack of investigation of inter- and intraobserver agreement variations limits the power of this study. In this study, we did focus on availability of CAD with different thresholds. Third, tumor size measurements were taken by only CADstream in our institution and the possibility of different results when using other CAD program cannot be excluded. However, both CADstream and DynaCAD (Invivo corp, Orlando, FL), which are the two most widely used CAD programs among all currently available systems, display the suspicious tissues based on contrast enhancement level above a set threshold in a similar way [13, 35-37]. Fourth, we did not investigate the yield of false-positive pixel predictions when using the 30\% threshold, although it is known that low threshold values can produce many false-positive pixel predictions of breast lesions. Lastly, we used three representative thresholds such as 30, 50 and 100\%, which are commonly used in clinical practice. However, the possibility of presence of other appropriate thresholds less than $50 \%$ cannot be excluded.

\section{Conclusion}

In conclusion, $\mathrm{CAD}$ is as accurate as manual measurement on MRI for preoperative tumor size estimation in breast cancer patients. Among the 3 most commonly used thresholds, $30 \%$ seems to be the most appropriate threshold and produced results similar to those of manual measurement on MRI for both invasive cancers and 
DCIS. However, we note a higher risk of underestimation for CAD with the 50 and $100 \%$ thresholds for invasive cancers and with the $100 \%$ threshold for DCIS. Our data analysis may help contribute to the consensus about the proper use of MRI and CAD for preoperative staging work-up in breast cancer patients.

\section{Abbreviations}

CAD: Computer-aided detection; MRI: Magnetic resonance imaging:

DCIS: Ductal carcinoma in situ; NME: Non-mass enhancement

\section{Acknowledgements}

Not applicable.

\section{Authors' contribution}

BKS made substantial contributions to the conception design of the work. SES was a major contributor in writing the manuscript. KRC, OHW, and EKP substantively revised the manuscipt. JC analyzed the data. SH created figures using data. All authors read and approved the final manuscript

\section{Funding}

Bo Kyoung Seo received National Research Foundation of Korea (NRF) grant funded by the Korea government (MSIP; Ministry of Science, ICT \& Future Planning) (No. NRF-2017R1A2B4010178).

\section{Availability of data and materials}

Please contact authors for data requests.

\section{Ethics approval and consent to participate}

This retrospective study was approved by institutional review board of the hospital (IRB No.2017AS0037).

\section{Consent for publication}

Written informed consent was obtained from each patient.

\section{Competing interests}

The authors declare that they have no competing interests.

\section{Author details}

'Department of Radiology, Korea University Anam Hospital, Korea University College of Medicine, 73 Goryeodae-ro, Seongbuk-gu, Seoul 02841, Republic of Korea. ${ }^{2}$ Department of Radiology, Korea University Ansan Hospital, Korea University College of Medicine, 123 Jeokgeum-ro, Danwon-gu, Ansan-si, Gyeonggi-do 15355, Republic of Korea. ${ }^{3}$ Department of Radiology, Korea University Guro Hospital, Korea University College of Medicine, 148 Gurodong-ro, Guro-gu, Seoul 08308, Republic of Korea. ${ }^{4}$ Medical Science Research Center, Korea University Ansan Hospital, 123 Jeokgeum-ro, Danwon-gu, Ansan-si, Gyeonggi-do 15355, Republic of Korea. ${ }^{5}$ Division of Clinical Bioinformatics, Biomedical Research Institute, Seoul National University Hospital, 101 Daehak-ro, Jongno-gu, Seoul 03080, Republic of Korea.

Received: 16 December 2019 Accepted: 14 April 2020 Published online: 28 April 2020

\section{References}

1. Smitt MC, Nowels KW, Zdeblick MJ, et al. The importance of the lumpectomy surgical margin status in long-term results of breast conservation. Cancer. 1995;76(2):259-67.

2. Gage I, Schnitt SJ, Nixon AJ, et al. Pathologic margin involvement and the risk of recurrence in patients treated with breast-conserving therapy. Cancer. 1996;78(9):1921-8.

3. Mullen R, Macaskill EJ, Khalil A, et al. Involved anterior margins after breast conserving surgery: is re-excision required? Eur J Surg Oncol. 2012;38(4): 302-6.

4. Uematsu T, Yuen S, Kasami M, et al. Comparison of magnetic resonance imaging, multidetector row computed tomography, ultrasonography, and mammography for tumor extension of breast cancer. Breast Cancer Res Treat. 2008;112(3):461-74.
5. Berg WA, Gutierrez L, NessAiver MS, et al. Diagnostic accuracy of mammography, clinical examination, US, and MR imaging in preoperative assessment of breast cancer. Radiology. 2004;233(3):830-49.

6. Shin HC, Han W, Moon HG, et al. Limited value and utility of breast MRI in patients undergoing breast-conserving cancer surgery. Ann Surg Oncol. 2012;19(8):2572-9.

7. Daniel OK, Lim SM, Kim JH, et al. Preoperative prediction of the size of pure ductal carcinoma in situ using three imaging modalities as compared to histopathological size: does magnetic resonance imaging add value? Breast Cancer Res Treat. 2017;164(2):437-44.

8. Song SE, Seo BK, Cho KR, et al. Computer-aided detection (CAD) system for breast MRI in assessment of local tumor extent, nodal status, and multifocality of invasive breast cancers: preliminary study. Cancer Imaging. 2015;15:1.

9. Rominger M, Berg D, Frauenfelder T, et al. Which factors influence MRIpathology concordance of tumour size measurements in breast cancer? Eur Radiol. 2016;26(5):1457-65

10. Houssami N, Ciatto S, Macaskill P, et al. Accuracy and surgical impact of magnetic resonance imaging in breast cancer staging: systematic review and meta-analysis in detection of multifocal and multicentric cancer. J Clin Oncol. 2008;26(19):3248-58.

11. Lai HW, Chen DR, Wu YC, et al. Comparison of the diagnostic accuracy of magnetic resonance imaging with Sonography in the prediction of breast Cancer tumor size: a concordance analysis with Histopathologically determined tumor size. Ann Surg Oncol. 2015;22(12):3816-23.

12. Beresford MJ, Padhani AR, Taylor NJ, et al. Inter- and intraobserver variability in the evaluation of dynamic breast cancer MRI. J Magn Reson Imaging. 2006:24(6):1316-25.

13. Dorrius MD, Jansen-van der Weide MC, van Ooijen PM, et al. Computeraided detection in breast MRI: a systematic review and meta-analysis. Eur Radiol. 2011:21:1600-8

14. Levman JE, Causer P, Warner $E$, et al. Effect of the enhancement threshold on the computer-aided detection of breast cancer using MRI. Acad Radiol. 2009;16:1064-9.

15. Lehman CD, Peacock S, DeMartini WB, et al. A new automated software system to evaluate breast MR examinations: improved specificity without decreased sensitivity. AJR Am J Roentgenol. 2006:187:51-6.

16. Williams TC, DeMartini WB, Partridge SC, et al. Breast MR imaging: computer-aided evaluation program for discriminating benign from malignant lesions. Radiology. 2007;244:94-103.

17. Baltzer PA, Renz DM, Kullnig PE, et al. Application of computer-aided diagnosis (CAD) in MR-mammography (MRM): do we really need whole lesion time curve distribution analysis? Acad Radiol. 2009;16(4):435-42.

18. Baltzer PA, Freiberg C, Beger S, et al. Clinical MR-mammography: are computer-assisted methods superior to visual or manual measurements for curve type analysis? A systematic approach. Acad Radiol. 2009;16(9):1070-6.

19. Arazi-Kleinman T, Causer PA, Jong RA, et al. Can breast MRI computer-aided detection (CAD) improve radiologist accuracy for lesions detected at MRI screening and recommended for biopsy in a high-risk population? Clin Radiol. 2009:64(12):1166-74.

20. Meeuwis C, van de Ven SM, Stapper G, et al. Computer-aided detection (CAD) for breast MRI: evaluation of efficacy at 3.0 T. Eur Radiol. 2010;20(3):522-8.

21. Morris EA, Comstock CE, Lee $\mathrm{CH}$. ACR BI-RADS magnetic resonance imaging In: CJ D'O, Sickles EA, Mendelson EB, Morris EA, editors. American College of Radiology (eds) ACR BI-RADS atlas, breast imaging reporting and data system. Reston, VA: American College of Radiology; 2013.

22. Hammond ME, Hayes DF, Dowsett M, et al. American Society of Clinical Oncology/College of American Pathologists guideline recommendations for immunohistochemical testing of estrogen and progesterone receptors in breast cancer. Arch Pathol Lab Med. 2010;134(6):907-22.

23. Lester SC, Connolly JL, Amin MB. College of American Pathologists protocol for the reporting of ductal carcinoma in situ. Arch Pathol Lab Med. 2009; 133(1):13-4.

24. Onesti JK, Mangus BE, Helmer SD, et al. Breast cancer tumor size: correlation between magnetic resonance imaging and pathology measurements. Am J Surg. 2008;196(6):844-8.

25. Boetes C, Mus RD, Holland R, et al. Breast tumors: comparative accuracy of MR imaging relative to mammography and US for demonstrating extent. Radiology. 1995;197(3):743-7.

26. Kristoffersen Wiberg M, Aspelin P, et al. Comparison of lesion size estimated by dynamic MR imaging, mammography and histopathology in breast neoplasms. Eur Radiol. 2003;13(6):1207-12. 
27. Demartini WB, Lehman CD, Peacock $S$, et al. Computer-aided detection applied to breast MRI: assessment of CAD-generated enhancement and tumor sizes in breast cancers before and after neoadjuvant chemotherapy. Acad Radiol. 2005:12(7):806-14.

28. Lyou CY, Cho N, Kim SM, et al. Computer-aided evaluation of breast MRI for the residual tumor extent and response monitoring in breast cancer patients receiving neoadjuvant chemotherapy. Korean J Radiol. 2011;12(1): 34-43.

29. Kim DY, Moon WK, Cho N, et al. MRI of the breast for the detection and assessment of the size of ductal carcinoma in situ. Korean J Radiol. 2007; 8(1):32-9.

30. Raza S, Vallejo M, Chikarmane SA, et al. Pure ductal carcinoma in situ: a range of MRI features. AJR Am J Roentgenol. 2008;191:689-99.

31. Jansen SA, Newstead GM, Abe H, et al. Pure ductal carcinoma in situ: kinetic and morphologic MR characteristics compared with mammographic appearance and nuclear grade. Radiology. 2007;245(3):684-91.

32. Kim JA, Son EJ, Youk JH, et al. MRl findings of pure ductal carcinoma in situ: kinetic characteristics compared according to lesion type and histopathologic factors. AJR Am J Roentgenol. 2011;196:1450-6.

33. Jiang YZ, Xia C, Peng WT, et al. Preoperative measurement of breast cancer overestimates tumor size compared to pathological measurement. PLoS One. 2014;9(1):e86676. https://doi.org/10.1371/journal.pone.0086676 eCollection 2014.

34. Mann RM, Veltman J, Barentsz JO, et al. The value of MRI compared to mammography in the assessment of tumour extent in invasive lobular carcinoma of the breast. Eur J Surg Oncol. 2008:34(2):135-42.

35. Yabuuchi H, Matsuo Y, Okafuji T, et al. Enhanced mass on contrastenhanced breast MR imaging: lesion characterization using combination of dynamic contrast-enhanced and diffusionweighted MR images. J Magn Reson Imaging. 2008;28(5):1157-65.

36. Turnbull LW. Dynamic contrast-enhanced MRI in the diagnosis and management of breast cancer. NMR Biomed. 2009;22(1):28-39.

37. Hauth EA, Jaeger $H$, Maderwald $S$, et al. Evaluation of quantitative parametric analysis for characterization of breast lesions in contrast enhanced MR mammography. Eur Radiol. 2006;16(12):2834-284.

\section{Publisher's Note}

Springer Nature remains neutral with regard to jurisdictional claims in published maps and institutional affiliations.

Ready to submit your research? Choose BMC and benefit from:

- fast, convenient online submission

- thorough peer review by experienced researchers in your field

- rapid publication on acceptance

- support for research data, including large and complex data types

- gold Open Access which fosters wider collaboration and increased citations

- maximum visibility for your research: over $100 \mathrm{M}$ website views per year

At $\mathrm{BMC}$, research is always in progress.

Learn more biomedcentral.com/submissions 